International Journal of Uncertainty, Fuzziness and Knowledge-Based Systems Vol. 15 , No. 3 (2007) 345-361

(C) World Scientific Publishing Company

\title{
REPRESENTABILITY IN INTERVAL-VALUED FUZZY SET THEORY
}

\author{
GLAD DESCHRIJVER* ${ }^{*}$ and CHRIS CORNELIS ${ }^{\dagger}$ \\ Department of Applied Mathematics and Computer Science, \\ Ghent University, Krijgslaan 281 (S9), B-9000 Gent, Belgium \\ *Glad.Deschrijver@UGent.be \\ $\dagger$ Chris.Cornelis@UGent.be \\ http://www.fuzzy.UGent.be
}

Received 26 January 2006

Revised 17 April 2007

\begin{abstract}
Interval-valued fuzzy set theory is an increasingly popular extension of fuzzy set theory where traditional [0,1]-valued membership degrees are replaced by intervals in $[0,1]$ that approximate the (unknown) membership degrees. To construct suitable graded logical connectives in this extended setting, it is both natural and appropriate to "reuse" ingredients from classical fuzzy set theory. In this paper, we compare different ways of representing operations on interval-valued fuzzy sets by corresponding operations on fuzzy sets, study their intuitive semantics, and relate them to an existing, purely ordertheoretical approach. Our approach reveals, amongst others, that subtle differences in the representation method can have a major impact on the properties satisfied by the generated operations, and that contrary to popular perception, interval-valued fuzzy set theory hardly corresponds to a mere twofold application of fuzzy set theory. In this way, by making the mathematical machinery behind the interval-valued fuzzy set model fully transparent, we aim to foster new avenues for its exploitation by offering application developers a much more powerful and elaborate mathematical toolbox than existed before.
\end{abstract}

Keywords: Interval-valued fuzzy sets; $\mathcal{L}$-fuzzy sets; graded logical connectives; representability.

\section{Introduction}

In 1965, Zadeh published his seminal work ${ }^{1}$ embodying the commonsense observation that real "objects" can possess a given property to a certain degree; therefore, the property is described by a $[0,1]$-valued membership function attributing to all objects a degree of membership in a fuzzy set. Since that time, the formalism has opened up a vast array of opportunities to intelligent control applications, flexible decision aid systems, approximate reasoning engines, ... As a tool for modelling vagueness, fuzzy logic has become synonymous with high-quality, low-cost solutions to problems whose inherent complexity defies the use of exact, "crisp" 
methods. While the sky seems to be the limit, it is clear at the same time both from theoretical observations and from an awareness of realistic application needs, that if fuzzy set theory wants to pursue its trendsetting role in dealing with imprecise knowledge, proper extensions of the original concept should be considered. For instance, within the current information society, embodied by the World Wide Web (WWW), robust tools are needed for dealing flexibly with facets of incompleteness and inconsistency (i.e., missing and contradictory information) that haunt a lot of typical data collections.

Many generalizations of fuzzy sets are available. For instance, membership degrees can be drawn not just from the unit interval $[0,1]$, but more generally from any complete lattice $\mathcal{L}$, as happens in Goguen's $\mathcal{L}$-fuzzy sets ${ }^{2}$; alternatively, they can be fixed only partially e.g. by specifying subsets of the evaluation set to which the exact membership degree is supposed to belong (see e.g. Gargov ${ }^{3}$ ). Going one step further, we can even allow fuzzy sets in $[0,1]$ as membership degrees: this is the setting of type 2 fuzzy set theory (see e.g. John ${ }^{4}$ ). Still another approach is to consider apart from the membership degree also a not necessarily complementary degree of non-membership, as is the practice in Atanassov's intuitionistic fuzzy set theory $^{5}$ as well as in bilattice theory (see e.g. Arieli et al. ${ }^{6}$ ).

Interval-valued fuzzy sets, which were apparently first studied by Sambuc $^{7}$ and which are characterized by a couple $\left(\mu_{A}^{l}, \mu_{A}^{r}\right)$ of a left and a right membership function such that $\mu_{A}^{l} \subseteq \mu_{A}^{r}$, take a very special place among these extensions. Formally, they are a special case of both $\mathcal{L}$-fuzzy sets $^{8}$ and of type 2 fuzzy sets (called "interval type 2 fuzzy sets" in that context, see e.g. Mendel $^{9}$ ) and they are even equivalent to intuitionistic fuzzy $\operatorname{sets}^{10}$. Moreover, they are straightforward to interpret: they serve to capture a feature of uncertainty w.r.t. the assignment of membership degrees in that the intervals may be understood to contain the true, incompletely known membership degree. Also, their manipulation does not pose the same computational barriers as encountered with general type 2 fuzzy sets.

However, this perceived simplicity is also rather deceptive. Most authors (and, indeed, most practical applications) represent operations on interval-valued fuzzy sets simply by a twofold application of a corresponding fuzzy set operation (i.e., applied once to the left and once to the right membership function, see e.g. Gehrke et al. ${ }^{8}$ and Jenei ${ }^{11}$ ). While there is definitely something to say in favour of this simplest and most intuitive approach, it reveals only a small tip of the iceberg while much more remains hidden beneath the surface. Recent work ${ }^{\mathrm{a}}$ has indicated the existence of operations not satisfying the mentioned representation, and has even demonstrated, in some cases, their superiority over the usual ones; some of them have several extremely useful properties which the representable operations can never meet. This observation calls for our closer attention. Is the sacrifice of these properties a price worth paying to preserve the illusion that one is working with

${ }^{\mathrm{a}}$ In particular, an order-theoretical exposition to the definition of graded logical connectives in interval-valued/intuitionistic fuzzy set theory (see e.g. Cornelis et al. ${ }^{12}$ and Deschrijver et al. ${ }^{13}$ ). 
parallel and independent applications of classical fuzzy set concepts? We believe not. Incidentally, as we will show in the remainder of this paper, most of the important "non-representable" operations, which may seem complicated at first glance, can be reduced to prototypical forms which still have a corresponding $[0,1]$-valued operation at their basis. Concretely, we present and evaluate a comprehensive typology of representation methods for graded logical connectives. This typology aims to make people more aware of the available alternatives and their respective semantics, and hence to foster new avenues of exploitation for the interval-valued fuzzy set model by offering application developers a more powerful and elaborate toolbox than existed before.

The rest of this paper is organized as follows: Section 2 provides some important background material regarding $\mathcal{L}$-fuzzy sets in general and interval-valued fuzzy sets in particular. After covering interval-valued negators in Section 3.1, Section 3.2 reviews and interprets the most important representation methods for t-norms and t-conorms in interval-valued fuzzy set theory. The representation of interval-valued implicators (which is influenced by interval-valued t-(co)norms as well as by implicators on $([0,1], \leq))$ is covered in Section 3.3, while in Section 3.4 we evaluate the various representation schemes w.r.t. desirable properties inspired by $\mathcal{L}$-fuzzy set theory. In Section 4, we round up the paper with some concluding remarks.

\section{Preliminary Definitions}

\section{1. $\mathcal{L}$-fuzzy set theory}

We set the scene by recalling the definition of $\mathcal{L}$-fuzzy sets, which will serve as the mathematical basis for an order-theoretical development of interval-valued fuzzy sets (henceforth abbreviated to IVFSs).

Definition 1. ( $\mathcal{L}$-fuzzy set $)^{2}$ Let $\mathcal{L}=\left(L, \leq_{L}\right)$ be a complete lattice, and $U$ a non-empty set called universe. An $\mathcal{L}$-fuzzy set in $U$ is defined as a $U \rightarrow L$ mapping.

For each $u$ in $U, A(u)$ represents the degree (in $L$ ) to which $u$ satisfies $A$. If, for $u, v$ in $U$ it holds that $A(u) \leq_{L} A(v)$, we say that $v$ satisfies $A$ at least as much as $u$. Sometimes neither $A(u) \leq_{L} A(v)$ nor $A(v) \leq_{L} A(u)$; in that case, we say that $u$ and $v$ are incomparable w.r.t. $A$ and write $A(u) \|_{L} A(v)$.

When $\mathcal{L}=([0,1], \leq)$, where $\leq$ represents the usual order on the unit interval, it is clear that we obtain classical fuzzy sets. Many important concepts in fuzzy set theory are defined in terms of the order $\leq$ on $[0,1]$, so it comes as no surprise that these concepts have straightforward extensions to $\mathcal{L}$-fuzzy sets. The most relevant for our purposes are graded logical connectives, which model, respectively, negation, conjunction, disjunction and implication. ${ }^{\mathrm{b}}$

${ }^{\mathrm{b}}$ From a set-theoretical perspective, the first three model the complement, intersection and union of $\mathcal{L}$-fuzzy sets. 
Definition 2. (Connectives in $\mathcal{L}$-fuzzy set theory) Let $\mathcal{L}=\left(L, \leq_{L}\right)$ be a complete lattice, such that $0_{\mathcal{L}}$ and $1_{\mathcal{L}}$ are the smallest, resp. greatest, elements of $\mathcal{L}$.

- A negator on $\mathcal{L}$ is an $\mathcal{L}$-decreasing $L \rightarrow L$ mapping $\mathcal{N}$ that satisfies $\mathcal{N}\left(0_{\mathcal{L}}\right)=1_{\mathcal{L}}$ and $\mathcal{N}\left(1_{\mathcal{L}}\right)=0_{\mathcal{L}}$. It is involutive if $\mathcal{N}(\mathcal{N}(x))=x$ for all $x$ in $L$.

- A $t$-norm on $\mathcal{L}$ is an $\mathcal{L}$-increasing, commutative, associative $L^{2} \rightarrow L$ mapping $\mathcal{T}$ that satisfies $\mathcal{T}\left(1_{\mathcal{L}}, x\right)=x$ for all $x$ in $L$.

- A $t$-conorm on $\mathcal{L}$ is an $\mathcal{L}$-increasing, commutative, associative $L^{2} \rightarrow L$ mapping $\mathcal{S}$ that satisfies $\mathcal{S}\left(0_{\mathcal{L}}, x\right)=x$ for all $x$ in $L$.

- An implicator on $\mathcal{L}$ is an $L^{2} \rightarrow L$ mapping $\mathcal{I}$ that is $\mathcal{L}$-decreasing in its first, and $\mathcal{L}$-increasing in its second component, and that satisfies $\mathcal{I}\left(1_{\mathcal{L}}, x\right)=x$ and $\mathcal{I}\left(0_{\mathcal{L}}, x\right)=1_{\mathcal{L}}$ for all $x$ in $L$.

It is clear that each member of the thus defined classes of connectives is a faithful extension of its counterpart from classical, two-valued logic. Which particular member to choose for a particular application depends on the specific properties required of the extension.

Based on de Morgan laws, t-norms and t-conorms can be related through an involutive negator $\mathcal{N}$ on $\mathcal{L}$; indeed, to each t-norm $\mathcal{T}$ on $\mathcal{L}$ corresponds its $\mathcal{N}$-dual t-conorm $\mathcal{T}^{\mathcal{N}}$ defined by, for $x, y$ in $L, \mathcal{T}^{\mathcal{N}}(x, y)=\mathcal{N}(\mathcal{T}(\mathcal{N}(x), \mathcal{N}(y)))$, and vice versa. Implicators are often derived from the other types of connectives. For our purposes, we consider $\mathrm{S}$ - and R-implicators: let $\mathcal{T}$ be a t-norm, $\mathcal{S}$ a t-conorm and $\mathcal{N}$ a negator on $\mathcal{L}$. Then the S-implicator $\mathcal{I}_{\mathcal{S}, \mathcal{N}}$ and the R-implicator $\mathcal{I}_{\mathcal{T}}$ are defined by, for $x, y$ in $L$,

$$
\begin{aligned}
& \mathcal{I}_{\mathcal{S}}(x, y)=\mathcal{S}(\mathcal{N}(x), y), \\
& \mathcal{I}_{\mathcal{T}}(x, y)=\sup \left\{\gamma \mid \gamma \in L \text { and } \mathcal{T}(x, \gamma) \leq_{L} y\right\}
\end{aligned}
$$

We say that a t-norm $\mathcal{T}$ on $\mathcal{L}$ satisfies the residuation principle if, for all $x, y, z$ in $L$

$$
\mathcal{T}(x, y) \leq_{L} z \Leftrightarrow y \leq_{L} \mathcal{I}_{\mathcal{T}}(x, z) .
$$

The residuation principle, which allows to construct a residuated lattice on top of $\mathcal{L}$, is of quintessential importance to approximate reasoning and formal deduction applications. It is well known that a t-norm $\mathcal{T}$ can only satisfy the residuation principle if the implicator involved is the R-implicator generated by $\mathcal{T}{ }^{14}$

\subsection{Interval-valued fuzzy set theory}

When we define

$$
\begin{aligned}
& L^{I}=\left\{\left[x_{1}, x_{2}\right] \mid\left(x_{1}, x_{2}\right) \in[0,1]^{2} \text { and } x_{1} \leq x_{2}\right\} \\
& {\left[x_{1}, x_{2}\right] \leq_{L^{I}}\left[y_{1}, y_{2}\right] \Leftrightarrow\left(x_{1} \leq y_{1} \text { and } x_{2} \leq y_{2}\right)}
\end{aligned}
$$

it is easy to verify that $\mathcal{L}^{I}=\left(L^{I}, \leq_{L^{I}}\right)$ is a complete lattice, and also that the class of IVFSs is isomorphic to that of $\mathcal{L}^{I}$-fuzzy sets. Hence, an IVFS $\left(\mu_{A}^{l}, \mu_{A}^{r}\right)$ 
emerges, syntactically, as a specific kind of $\mathcal{L}$-fuzzy set, in the sense that, for every $u$ in $U,\left[\mu_{A}^{l}(u), \mu_{A}^{r}(u)\right] \in \mathcal{L}^{I}$. Thus, the lattice $\mathcal{L}^{I}$ gives us an elegant and compact environment in which to calculate with IVFSs. A graphical representation of $\mathcal{L}^{I}$ is shown in Figure 1. The smallest element of the lattice is $0_{\mathcal{L}^{I}}=[0,0]$, the greatest $1_{\mathcal{L}^{I}}=[1,1]$. The hypothenuse $D$ of the triangle in Figure 1 contains the elements $x=\left[x_{1}, x_{2}\right]$ of $L^{I}$ such that $x_{1}=x_{2}$. Hence, $D$ represents the values in $L^{I}$ about which there is no indeterminacy and can be identified with the unit interval $[0,1]$ from (classical) fuzzy set theory.

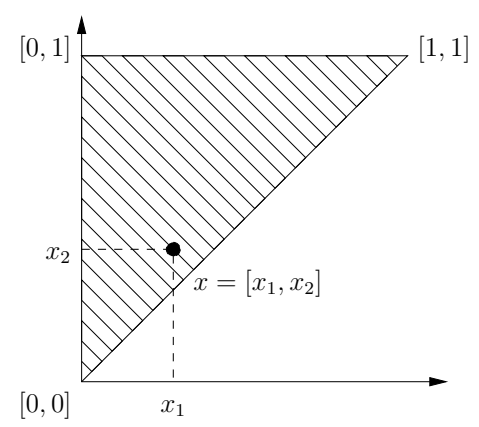

Fig. 1. The lattice $\mathcal{L}^{I}$.

By virtue of the interval order $\leq_{L_{I}}$, graded logical connectives in IVFS theory can be constructed as instances of Definition 2. By contrast, it is also possible to generate connectives by applying $[0,1]$-valued operations to the left, resp. right membership functions $\mu^{l}$ and $\mu^{r}$, which are, after all, fuzzy sets. In Section 3, we will put these two approaches side by side and unravel their relationship. As a tool for guiding our analysis, we will frequently use so-called extended modal operators (after Atanassov $\left.{ }^{15}\right)$ :

Definition 3. (Extended modal operators) Let $\alpha \in[0,1]$. Then the extended modal operator $D_{\alpha}$ is an $L^{I} \rightarrow[0,1]$ mapping defined by, for $x=\left[x_{1}, x_{2}\right]$ in $L^{I}$,

$$
D_{\alpha} x=x_{1}+\alpha\left(x_{2}-x_{1}\right)
$$

Extended modal operators are in particular useful for transforming an IVFS into a fuzzy set. Intuitively, the interval $\left[\mu_{A}^{l}(u), \mu_{A}^{r}(u)\right]$ represents an array of possible values for the "real" membership degree of $u$ to $A$, and $\alpha$ acts a parameter of optimism in resolving that indeterminacy. Clearly, $D_{0} A(u)=\mu_{A}^{l}(u)$ (minimal membership) and $D_{1} A(u)=\mu_{A}^{r}(u)$ (maximal membership) represent the two extremes w.r.t. the transformation. In the sequel, after Atanassov ${ }^{15}$, we will also denote, for $x=\left[x_{1}, x_{2}\right]$ in $L^{I}, D_{0} x=x_{1}$ by $\square x$ (necessity) and $D_{1} x=x_{2}$ by $\diamond x$ (possibility). 


\section{Representation Methods for Graded Logical Connectives}

In this section, we discuss in detail generation techniques for IVFS connectives, using $[0,1]$-valued ingredients and extended modal operators as our key tools. Our first and foremost goal is to shine a bright light on what has been achieved in earlier works, and to convey a kind of "IVFS literacy" to a general audience interested in working with IVFSs. Hence, in order not to harm the clarity of the exposition, the emphasis is not necessarily on the most general representation methods, but rather on the most practically useful ones. For the interested reader wishing to learn more about the precise mathematical details, technical notes and/or references to the literature are provided.

Negators, briefly covered in Section 3.1, are our first focus. The pivotal constructs in this study, however, are t-norms and t-conorms (Section 3.2). Implicators, as "derived" connectives, are studied in Section 3.3, while a critical evaluation of the presented representation methods w.r.t induced properties is given in Section 3.4. For ease of notation, in this section we assume that an element $x$ of $L^{I}$ is always of the form $\left[x_{1}, x_{2}\right]$.

\subsection{Representation of negators}

From the point of view of representation, negators are the simplest and most straightforward kind of connective, so their treatment serves as a very gentle introduction to the main exposition. Briefly, given a negator $N$ on $([0,1], \leq)$, it is easy to verify that the mapping $\mathcal{N}_{N}: L^{I} \rightarrow L^{I}$ defined by, for all $x$ in $L^{I}$,

$$
\mathcal{N}_{N}(x)=\left[N\left(x_{2}\right), N\left(x_{1}\right)\right]=[N(\diamond x), N(\square x)]
$$

is a negator on $\mathcal{L}^{I}$. Moreover, since for each $\alpha$ in $[0,1], N(\diamond x) \leq N\left(D_{\alpha} x\right) \leq N(\square x)$, it holds that

$$
\left\{N\left(D_{\alpha} x\right) \mid \alpha \in[0,1]\right\} \subseteq \mathcal{N}_{N}(x),
$$

so it is justified to say that the interval $\mathcal{N}_{N}(x)$ "includes all possible situations that could occur if the uncertainty in $x$ were to be expelled." Note that if $N$ is continuous, then from the mean-value theorem follows that equality holds in (2).

Definition 4. A negator $\mathcal{N}$ on $\mathcal{L}^{I}$ is called $n$-representable if there exists a negator $N$ on $([0,1], \leq)$ such that $\mathcal{N}=\mathcal{N}_{N}$, where $\mathcal{N}_{N}$ is given by (1). The negator $N$ is then called the representative of $\mathcal{N}$.

Note 1. In a more general setting, $\left[N\left(x_{2}\right), N\left(x_{1}\right)\right]$ can be replaced by $\left[N_{1}\left(x_{2}\right), N_{2}\left(x_{1}\right)\right]$, where $N_{1}$ and $N_{2}$ are negators on $([0,1], \leq)$ such that $N_{1} \leq N_{2}$.

Example 1. The standard negator $\mathcal{N}_{s}$ on $\mathcal{L}^{I}$, given by $\mathcal{N}_{s}(x)=\left[1-x_{2}, 1-x_{1}\right]$, is n-representable. This does not hold in general; take for instance $\mathcal{N}$ defined by, for all $x$ in $L^{I}$,

$$
\mathcal{N}(x)= \begin{cases}1_{\mathcal{L}^{I}}, & \text { if } x=0_{\mathcal{L}^{I}}, \\ 0_{\mathcal{L}^{I}}, & \text { otherwise }\end{cases}
$$


The following proposition recalls an important result about the representation of involutive negators on $\mathcal{L}^{I}$ which will be needed in the sequel:

Proposition 1. ${ }^{13}$ A negator $\mathcal{N}$ on $\mathcal{L}^{I}$ is involutive if and only if there exists an involutive negator $N$ on $([0,1], \leq)$ such that $\mathcal{N}=\mathcal{N}_{N}$.

Note 2. This representation is a special case of a more general result ${ }^{8}$ that shows that all anti-automorphisms of $\mathcal{L}^{I}$ are representable.

\subsection{Representation of t-norms and t-conorms}

\subsection{1. t-Representability}

Given a t-norm $T$ on $([0,1], \leq)$, a straightforward way to generate a corresponding connective on $\mathcal{L}^{I}$ is by defining, for $x, y$ in $L^{I}$ (see also Gehrke et al. ${ }^{8}$ and Jenei ${ }^{11}$ )

$$
\mathcal{T}_{T}(x, y)=\left[T\left(x_{1}, y_{1}\right), T\left(x_{2}, y_{2}\right)\right]=[T(\square x, \square y), T(\diamond x, \diamond y)] .
$$

Since it holds that for all $\alpha, \beta$ in $[0,1], T(\square x, \square y) \leq T\left(D_{\alpha} x, D_{\beta} y\right) \leq T(\diamond x, \diamond y)$, it also holds, if $T$ is continuous, that

$$
\mathcal{T}_{T}(x, y)=\left\{T\left(D_{\alpha} x, D_{\beta} y\right) \mid \alpha, \beta \in[0,1]\right\} .
$$

Hence, the interval $\mathcal{T}_{T}(x, y)$ indeed includes all possible situations that can occur if the uncertainty vanishes from $x$ and $y$, just like we observed for $\mathcal{N}_{N}(x)$. Similarly, from a t-conorm $S$ on $([0,1], \leq)$ we can naturally construct an associated t-conorm $\mathcal{S}_{S}$ on $\mathcal{L}^{I}$ with an analogous interpretation.

Definition 5. (t-representability) Given a t-norm $T$ and a t-conorm $S$ on $([0,1], \leq)$, the $\left(L^{I}\right)^{2} \rightarrow L^{I}$ mappings $\mathcal{T}_{T}^{r}$ and $\mathcal{S}_{S}^{r}$ defined by, for $x, y$ in $L^{I}$,

$$
\begin{gathered}
\mathcal{T}_{T}^{r}(x, y)=\left[T\left(x_{1}, y_{1}\right), T\left(x_{2}, y_{2}\right)\right]=[T(\square x, \square y), T(\diamond x, \diamond y)], \\
\mathcal{S}_{S}^{r}(x, y)=\left[S\left(x_{1}, y_{1}\right), S\left(x_{2}, y_{2}\right)\right]=[S(\square x, \square y), S(\diamond x, \diamond y)] .
\end{gathered}
$$

are a t-norm, resp. a t-conorm on $\mathcal{L}^{I}$ that are called the t-representable t-norm (resp. t-conorm) on $\mathcal{L}^{I}$ with representative $T(\operatorname{resp} . S)^{\mathrm{c}}$.

\section{Example 2.}

- Let $T_{M}$ be the minimum t-norm on $([0,1], \leq)$, then $\mathcal{T}_{T_{M}}^{r}(x, y)=\left[\min \left(x_{1}, y_{1}\right)\right.$, $\left.\min \left(x_{2}, y_{2}\right)\right]$ for $x, y$ in $L^{I}$. Note that $\mathcal{T}_{T_{M}}^{r}$ equals the infimum on $\mathcal{L}^{I}$. Analogously, for $S_{M}$ the maximum t-conorm on $([0,1], \leq), \mathcal{S}_{S_{M}}^{r}$ equals the supremum on $\mathcal{L}^{I}$.

- Let $T_{W}$ be the Eukasiewicz t-norm on $([0,1], \leq)$, that is, $T_{W}(x, y)=\max (0, x+$ $y-1)$ for $x, y$ in $[0,1]$. Then $\mathcal{T}_{T_{W}}^{r}(x, y)=\left[\max \left(0, x_{1}+y_{1}-1\right), \max \left(0, x_{2}+y_{2}-1\right)\right]$, for $x, y$ in $L^{I}$. For notational ease, we abbreviate $\mathcal{T}_{T_{W}}^{r}$ to $\mathcal{T}_{W}^{r}$ and call it the trepresentable Łukasiewicz t-norm on $\mathcal{L}^{I}$. Analogously, for $S_{W}$ the Łukasiewicz

\footnotetext{
${ }^{\mathrm{c}} \mathrm{A}$ slightly more general definition of t-representability, using two different $\mathrm{t}$-(co)norms on ([0, 1],
} $\leq)$, appears in the work of Deschrijver et al. ${ }^{13}$ 
t-conorm on $([0,1], \leq)$, that is, $S_{W}(x, y)=\min (1, x+y)$ for $x, y$ in $[0,1], \mathcal{S}_{S_{W}}^{r}$ is abbreviated to $\mathcal{S}_{W}^{r}$ and called the t-representable Łukasiewicz t-conorm on $\mathcal{L}^{I}$.

\subsubsection{Pseudo-t-representability}

Not all t-norms on $\mathcal{L}^{I}$ are t-representable; this was first noted by Cornelis et al. ${ }^{16}$ Deschrijver et al. ${ }^{17}$ introduced a non-t-representable t-norm and its $\mathcal{N}_{s}$-dual tconorm which have interesting properties that no t-representable t-(co)norm satisfies. Deschrijver et al. ${ }^{13}$ and Deschrijver and Kerre ${ }^{18}$ generalized these observations by characterizing several classes of t-norms on $\mathcal{L}^{I}$ meeting various properties. The purpose of this paragraph is to investigate these classes from the point of view of representation by connectives on $([0,1], \leq)$.

Example 3. The non-t-representable t-norm $\mathcal{T}_{W}$ introduced by Cornelis et al. ${ }^{16}$ is defined by, for all $x, y$ in $L^{I}$,

$$
\begin{aligned}
\mathcal{T}_{W}(x, y) & =\left[\max \left(0, x_{1}+y_{1}-1\right), \max \left(0, x_{1}+y_{2}-1, x_{2}+y_{1}-1\right)\right] \\
& =\left[T_{W}(\square x, \square y), \max \left(T_{W}(\square x, \diamond y), T_{W}(\diamond x, \square y)\right)\right] .
\end{aligned}
$$

Looking at its structure, this t-norm has the same lower bound as the trepresentable Łukasiewicz t-norm $\mathcal{T}_{W}^{r}$, but differs from it by its upper bound: instead of taking the "optimum" value $T_{W}(\diamond x, \diamond y)$, the second component is obtained by taking the maximum of $T_{W}(\square x, \diamond y)$ and $T_{W}(\diamond x, \square y)$. Hence it is not guaranteed that the interval $\mathcal{T}_{W}(x, y)$ contains all possible values $T_{W}\left(D_{\alpha} x, D_{\beta} y\right)$ for $\alpha, \beta$ in $[0,1]$. Rather,

$$
\mathcal{T}_{W}(x, y)=\left\{T_{W}\left(D_{\alpha} x, \square y\right) \mid \alpha \in[0,1]\right\} \cup\left\{T_{W}\left(\square x, D_{\beta} y\right) \mid \beta \in[0,1]\right\} .
$$

What this representation enforces is that, in eliminating the uncertainty from $x$ and $y$, we have to impose for at least one of them the "worst" possible value $(\square x$, resp. $\square y$ ). Therefore, this could be called a pessimistic approach to the definition of a t-norm on $\mathcal{L}^{I}$. A dual phenomenon occurs for the $\mathcal{N}_{s}$-dual t-conorm $\mathcal{S}_{W}$ of $\mathcal{T}_{W}$, given by, for all $x, y$ in $L^{I}$,

$$
\begin{aligned}
\mathcal{S}_{W}(x, y) & =\left[\min \left(1, x_{1}+y_{2}, x_{2}+y_{1}\right), \min \left(1, x_{2}+y_{2}\right)\right] \\
& =\left[\min \left(S_{W}(\square x, \diamond y), S_{W}(\diamond x, \square y)\right), S_{W}(\diamond x, \diamond y)\right] .
\end{aligned}
$$

Here, the adapted lower bound reflects an optimistic inclination. Indeed,

$$
\mathcal{S}_{W}(x, y)=\left\{S_{W}\left(D_{\alpha} x, \diamond y\right) \mid \alpha \in[0,1]\right\} \cup\left\{T_{W}\left(\diamond x, D_{\beta} y\right) \mid \beta \in[0,1]\right\} .
$$

On the other hand, it can be verified that the formulas

$$
\begin{aligned}
& \mathcal{T}_{W}^{\prime}(x, y)=\left[\min \left(T_{W}(\square x, \diamond y), T_{W}(\diamond x, \square y)\right), T_{W}(\diamond x, \diamond y)\right], \\
& \mathcal{S}_{W}^{\prime}(x, y)=\left[S_{W}(\square x, \square y), \max \left(S_{W}(\square x, \diamond y), S_{W}(\diamond x, \square y)\right)\right],
\end{aligned}
$$

for $x, y$ in $L^{I}$, define a t-norm and a t-conorm on $\mathcal{L}^{I}$ that reflect optimism and pessimism, respectively. 
The above strategy can be turned into a productive mechanism for generating optimistic and pessimistic pseudo-t-representable t-norms and t-conorms on $\mathcal{L}^{I}$ from corresponding connectives on $([0,1], \leq)$. This is formalized by the following definition.

Definition 6. (Pseudo-t-representability) Given a t-norm $T$ and a t-conorm $S$ on $([0,1], \leq)$, the $\left(L^{I}\right)^{2} \rightarrow L^{I}$ mappings $\mathcal{T}_{T}^{p}$ and $\mathcal{S}_{S}^{p}$ defined by, for $x, y$ in $L^{I}$,

$$
\begin{aligned}
\mathcal{T}_{T}^{p}(x, y) & =\left[T\left(x_{1}, y_{1}\right), \max \left(T\left(x_{1}, y_{2}\right), T\left(x_{2}, y_{1}\right)\right)\right] \\
& =[T(\square x, \square y), \max (T(\square x, \diamond y), T(\diamond x, \square y))], \\
\mathcal{S}_{S}^{p}(x, y) & =\left[S\left(x_{1}, y_{1}\right), \max \left(S\left(x_{1}, y_{2}\right), S\left(x_{2}, y_{1}\right)\right)\right] \\
& =[S(\square x, \square y), \max (S(\square x, \diamond y), S(\diamond x, \square y))],
\end{aligned}
$$

are a t-norm, resp. a t-conorm on $\mathcal{L}^{I}$ that are called the pessimistic t-norm (resp. t-conorm) on $\mathcal{L}^{I}$ with representative $T$ (resp. $S$ ). Analogously, the $\left(L^{I}\right)^{2} \rightarrow L^{I}$ mappings $\mathcal{T}_{T}^{o}$ and $\mathcal{S}_{S}^{o}$ defined by, for $x, y$ in $L^{I}$,

$$
\begin{aligned}
\mathcal{T}_{T}^{o}(x, y) & =\left[\min \left(T\left(x_{1}, y_{2}\right), T\left(x_{2}, y_{1}\right)\right), T\left(x_{2}, y_{2}\right)\right] \\
& =[\min (T(\square x, \diamond y), T(\diamond x, \square y)), T(\diamond x, \diamond y)], \\
\mathcal{S}_{S}^{o}(x, y) & =\left[\min \left(S\left(x_{1}, y_{2}\right), S\left(x_{2}, y_{1}\right)\right), S\left(x_{2}, y_{2}\right)\right] \\
& =[\min (S(\square x, \diamond y), S(\diamond x, \square y)), S(\diamond x, \diamond y)],
\end{aligned}
$$

are a t-norm, resp. a t-conorm on $\mathcal{L}^{I}$ which are called the optimistic t-norm (resp. t-conorm) on $\mathcal{L}^{I}$ with representative $T$ (resp. $S$ ). A t-(co)norm on $\mathcal{L}^{I}$ is called pseudo-t-representable if it is either optimistic or pessimistic.

Note 3. A class of t-norms generalizing both the t-representable t-norms and the pessimistic t-norms can be introduced. Let $T$ be a t-norm on $([0,1], \leq)$, and $t \in[0,1]$. Then the mapping $\mathcal{T}_{T, t}:\left(L^{I}\right)^{2} \rightarrow L^{I}$ defined by, for all $x, y$ in $L^{I}$,

$$
\mathcal{T}_{T, t}(x, y)=\left[T\left(x_{1}, y_{1}\right), \max \left(T\left(t, T\left(x_{2}, y_{2}\right)\right), T\left(x_{1}, y_{2}\right), T\left(x_{2}, y_{1}\right)\right)\right]
$$

is a t-norm on $\mathcal{L}^{I}$. The usage of this class is, that it allows the user to define $\mathcal{T}([0,1],[0,1])=[0, t]$ arbitrarily. This can be useful in applications where in some situations one needs to impose that the conjunction of two completely unknown propositions is also unknown (e.g. "the sun will shine tomorrow" and "Olga has black hair"), while in other situations it would be more appropriate that the conjunction of two unknown statements is false (e.g. "Olga has blond hair" and "Olga has black hair"). If $t=0$, then we obtain the pessimistic t-norms, if $t=1$ then we find the t-representable t-norms. Clearly, since the lower bound of $\mathcal{T}_{T, t}$ is independent of $x_{2}$ and $y_{2}$, the optimistic t-norms $\mathcal{T}_{T}^{o}$ do not belong to this class as soon as $T \neq$ min. In Section 3.4.3 a more thorough discussion on possible extensions of (pseudo-)t-representable t-norms will be given. 
The following proposition relates the representability of a t-norm on $\mathcal{L}^{I}$ to the representability of its $\mathcal{N}$-dual. In a completely similar way the representability of a t-conorm on $\mathcal{L}^{I}$ can be related to the representability of its $\mathcal{N}$-dual.

Proposition 2. Let $\mathcal{T}_{T}^{r}$ be a t-representable t-norm on $\mathcal{L}^{I}$ with representative $T$, and let $\mathcal{N}_{N}$ be an involutive negator with representative $N$, then the $\mathcal{N}_{N}$-dual of $\mathcal{T}_{T}^{r}$ is the t-representable $t$-conorm on $\mathcal{L}^{I}$ with representative the $N$-dual of $T$, i.e.

$$
\left(\mathcal{T}_{T}^{r}\right)^{\mathcal{N}_{N}}=\mathcal{S}_{T^{N}}^{r}
$$

Let $\mathcal{T}_{T}^{p}$ be a pessimistic t-norm on $\mathcal{L}^{I}$ with representative $T$, and let $\mathcal{N}_{N}$ be an involutive negator with representative $N$, then the $\mathcal{N}_{N}$-dual of $\mathcal{T}_{T}^{p}$ is the optimistic t-conorm on $\mathcal{L}^{I}$ with representative the $N$-dual of $T$, i.e.

$$
\left(\mathcal{T}_{T}^{p}\right)^{\mathcal{N}_{N}}=\mathcal{S}_{T^{N}}^{o}
$$

Let $\mathcal{T}_{T}^{o}$ be an optimistic t-norm on $\mathcal{L}^{I}$ with representative $T$, and let $\mathcal{N}_{N}$ be an involutive negator with representative $N$, then the $\mathcal{N}_{N}$-dual of $\mathcal{T}_{T}^{o}$ is the pessimistic t-conorm on $\mathcal{L}^{I}$ with representative the $N$-dual of $T$, i.e.

$$
\left(\mathcal{T}_{T}^{o}\right)^{\mathcal{N}_{N}}=\mathcal{S}_{T^{N}}^{p}
$$

Proof. The first part is shown by Deschrijver et al. ${ }^{13}$ We prove the second part. The third part is proven similarly.

Let $\mathcal{T}_{T}^{p}$ be a pessimistic t-norm on $\mathcal{L}^{I}$ with representative $T$, and let $\mathcal{N}_{N}$ be an involutive negator with representative $N$ (such a representative exists by Proposition 1). Then, for all $x, y$ in $L^{I}$,

$$
\begin{aligned}
\left(\mathcal{T}_{T}^{p}\right)^{\mathcal{N}_{N}}(x, y) & =\mathcal{N}\left(\left[T\left(N\left(x_{2}\right), N\left(y_{2}\right)\right), \max \left(T\left(N\left(x_{2}\right), N\left(y_{1}\right)\right), T\left(N\left(x_{1}\right), N\left(y_{2}\right)\right)\right)\right]\right) \\
& =\left[\min \left(T^{N}\left(x_{2}, y_{1}\right), T^{N}\left(x_{1}, y_{2}\right)\right), T^{N}\left(x_{2}, y_{2}\right)\right] \\
& =\mathcal{S}_{T^{N}}^{o}(x, y) .
\end{aligned}
$$

\subsection{Representation of implicators}

Similarly as for t-(co)norms we can introduce a kind of direct representability for implicators on $\mathcal{L}^{I}$, as well as two kinds of pseudo-representability, by means of implicators on $([0,1], \leq)$.

\subsection{1. i-Representability}

Definition 7. (i-representability) ${ }^{\mathrm{d}}$ Given an implicator $I$ on $([0,1], \leq)$, the $\left(L^{I}\right)^{2} \rightarrow L^{I}$ mapping $\mathcal{I}_{I}^{r}$ defined by, for $x, y$ in $L^{I}$,

$$
\mathcal{I}_{I}^{r}(x, y)=\left[I\left(x_{2}, y_{1}\right), I\left(x_{1}, y_{2}\right)\right]=[I(\diamond x, \square y), I(\square x, \diamond y)]
$$

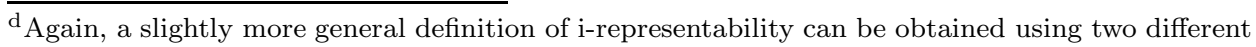
implicators $I_{1}$ and $I_{2}$ on $([0,1], \leq)$, such that $I_{1} \leq I_{2}$.
} 
is an implicator which is called the i-representable implicator on $\mathcal{L}^{I}$ with representative $I$.

By the fact that for continuous $\mathcal{I}_{I}^{r}$ it holds that

$$
\mathcal{I}_{I}^{r}(x, y)=\left\{I\left(D_{\alpha} x, D_{\beta} y\right) \mid \alpha, \beta \in[0,1]\right\},
$$

it is clear that i-representability is on a par with n-representability and trepresentability.

\subsubsection{Pseudo-i-representability}

Definition 8. (Pseudo-i-representability) Given an implicator $I$ on $([0,1], \leq)$, the $\left(L^{I}\right)^{2} \rightarrow L^{I}$ mappings $\mathcal{I}_{I}^{p}$ and $\mathcal{I}_{I}^{o}$ defined by, for $x, y$ in $L^{I}$,

$$
\begin{aligned}
\mathcal{I}_{I}^{p}(x, y) & =\left[I\left(x_{2}, y_{1}\right), \max \left(I\left(x_{1}, y_{1}\right), I\left(x_{2}, y_{2}\right)\right)\right] \\
& =[I(\diamond x, \square y), \max (I(\square x, \square y), I(\diamond x, \diamond y))], \\
\mathcal{I}_{I}^{o}(x, y) & =\left[\min \left(I\left(x_{1}, y_{1}\right), I\left(x_{2}, y_{2}\right)\right), I\left(x_{1}, y_{2}\right)\right] \\
& =[\min (I(\square x, \square y), I(\diamond x, \diamond y)), I(\square x, \diamond y)],
\end{aligned}
$$

are implicators on $\mathcal{L}^{I}$ which are called the pessimistic, resp. optimistic implicator on $\mathcal{L}^{I}$ with representative $I$. An implicator on $\mathcal{L}^{I}$ is called pseudo-i-representable if it is either optimistic or pessimistic.

\subsubsection{Links with $t$-(co)norms}

Implicators on $\mathcal{L}^{I}$ can also be generated in a different way: from t-(co)norms and negators as S-implicators and R-implicators. In this paragraph, we study the relationship of these constructs to i-representability and pseudo-i-representability.

For S-implicators and i-representability, the situation is rather straightforward and can be summed up by the following result which is shown (in a slightly more general form) by Baczyński. ${ }^{19}$

Proposition 3. A mapping $\mathcal{I}:\left(L^{I}\right)^{2} \rightarrow L^{I}$ is an S-implicator generated by a $t$-representable t-conorm and an involutive negator on $\mathcal{L}^{I}$ if and only if there exists an $S$-implicator $I_{S, N}$ generated by a t-conorm $S$ and an involutive negator $N$ on $([0,1], \leq)$ such that, for all $x, y$ in $L^{I}$,

$$
\mathcal{I}(x, y)=\left[I_{S, N}\left(x_{2}, y_{1}\right), I_{S, N}\left(x_{1}, y_{2}\right)\right]
$$

So, S-implicators on $\mathcal{L}^{I}$ generated by a t-representable t-conorm and an involutive negator are i-representable implicators having an S-implicator on $([0,1], \leq)$ as their representative. For R-implicators, no such transparent relation with irepresentability exists.

Proposition 4. No R-implicator on $\mathcal{L}^{I}$ is i-representable. 
We discuss now how optimistic and pessimistic implicators can be related to optimistic and pessimistic t-norms through the construction of the corresponding R- and S-implicators.

Proposition 5. Let $\mathcal{T}_{T}^{p}$ be a pessimistic t-norm on $\mathcal{L}^{I}$. Then the R-implicator generated by $\mathcal{T}_{T}^{p}$ is given by the optimistic implicator with representative $I_{T}$, i.e.

$$
\mathcal{I}_{\mathcal{T}_{T}^{p}}=\mathcal{I}_{I_{T}}^{o}
$$

Proposition 6. Let $\mathcal{T}_{T}^{o}$ be an optimistic t-norm on $\mathcal{L}^{I}$. Then its residual implicator is given by, for all $x, y$ in $L^{I}$,

$$
\mathcal{I}_{\mathcal{T}_{T}^{o}}(x, y)=\left[\min \left(I_{T}\left(x_{1}, y_{1}\right), I_{T}\left(x_{2}, y_{2}\right)\right), I_{T}\left(x_{2}, y_{2}\right)\right]
$$

This formula resembles the one corresponding to optimistic implicators. However, the upper bound involves $x_{2}$ instead of $x_{1}$, so contrary to optimistic implicators this bound does not correspond to the highest possible value of $I\left(D_{\alpha} x, D_{\beta} y\right)$, where $\alpha, \beta$ in $[0,1]$. Obviously, $\mathcal{I}_{\mathcal{T}_{T}^{o}}$ is not a pessimistic implicator either. Moreover, it is equal to the R-implicator generated by the corresponding t-representable t-norm.

Proposition 7. Let $\mathcal{T}_{T}^{r}$ be a t-representable t-norm on $\mathcal{L}^{I}$. Then its residual implicator is given by, for all $x, y$ in $L^{I}$,

$$
\mathcal{I}_{\mathcal{T}_{T}^{r}}(x, y)=\left[\min \left(I_{T}\left(x_{1}, y_{1}\right), I_{T}\left(x_{2}, y_{2}\right)\right), I_{T}\left(x_{2}, y_{2}\right)\right] .
$$

Deschrijver and Kerre ${ }^{18}$ have shown that if a t-norm $\mathcal{T}$ on $\mathcal{L}^{I}$ satisfies the residuation principle, then the first component of $\mathcal{T}(x, y)$ is independent of $x_{2}$ and $y_{2}$. From this follows:

Proposition 8. Optimistic t-norms for which the representative is not the minimum $t$-norm $T_{M}$ do not satisfy the residuation principle.

Proof. If $T \neq T_{M}$, then there exists an element $a \in[0,1]$ such that $T(a, a)<a$. Let $x=[a, a]$ and $x^{\prime}=y=[a, 1]$ be elements of $L^{I}$. Then we obtain for the optimistic t-norm with representative $T$ that $\square \mathcal{T}_{T}^{o}(x, y)=\min (a, T(a, a))=T(a, a)<a=$ $\square \mathcal{T}_{T}^{o}\left(x^{\prime}, y\right)$, so $\square \mathcal{T}_{T}^{o}(x, y)$ is not independent of $x_{2}$.

On the other hand, if $T$ is a left-continuous t-norm, then the t-representable tnorm $\mathcal{T}_{T}^{r}$ and the pessimistic t-norm $\mathcal{T}_{T}^{p}$ both do satisfy the residuation principle. ${ }^{18}$

For the S-implicators corresponding to pessimistic and optimistic t-conorms we obtain the following.

Proposition 9. Let $\mathcal{S}_{S}^{p}$ be a pessimistic t-conorm on $\mathcal{L}^{I}$ with representative $S$, and let $\mathcal{N}_{N}$ be an n-representable negator with representative $N$. Then the $S$-implicator generated by $\mathcal{S}_{S}^{p}$ and $\mathcal{N}_{N}$ is the pessimistic implicator with representative $I_{S, N}$, i.e.

$$
\mathcal{I}_{\mathcal{S}_{S}^{p}, \mathcal{N}_{N}}=\mathcal{I}_{I_{S, N}}^{p} .
$$


Let $\mathcal{S}_{S}^{o}$ be an optimistic t-conorm on $\mathcal{L}^{I}$ with representative $S$, and let $\mathcal{N}_{N}$ be an $n$-representable negator with representative $N$. Then the $S$-implicator generated by $\mathcal{S}_{S}^{o}$ and $\mathcal{N}_{N}$ is the optimistic implicator with representative $I_{S, N}$, i.e.

$$
\mathcal{I}_{\mathcal{S}_{S}^{o}, \mathcal{N}_{N}}=\mathcal{I}_{I_{S, N}}^{o}
$$

Proof. Let $\mathcal{S}_{S}^{p}$ be a pessimistic t-conorm on $\mathcal{L}^{I}$ with representative $S$, and let $\mathcal{N}_{N}$ be an n-representable negator with representative $N$. Then, for all $x, y$ in $L^{I}$,

$$
\begin{aligned}
\mathcal{I}_{\mathcal{S}_{S}^{p}, \mathcal{N}_{N}}(x, y) & =\mathcal{S}_{S}^{p}\left(\mathcal{N}_{N}(x), y\right) \\
& =\left[S\left(N\left(x_{2}\right), y_{1}\right), \max \left(S\left(N\left(x_{2}\right), y_{2}\right), S\left(N\left(x_{1}\right), y_{1}\right)\right)\right] \\
& =\left[I_{S, N}\left(x_{2}, y_{1}\right), \max \left(I_{S, N}\left(x_{2}, y_{2}\right), I_{S, N}\left(x_{1}, y_{1}\right)\right)\right] \\
& =\mathcal{I}_{I_{S, N}}^{p}(x, y) .
\end{aligned}
$$

The second part is proven similarly.

We see that pessimistic t-norms generate optimistic R-implicators, but optimistic t-norms do not generate pessimistic implicators. The R-implicators generated by optimistic t-norms coincide with the R-implicators generated by t-representable t-norms. However, no intuitive interpretation of these R-implicators can be given. On the other hand, for S-implicators the situation is clearer: pessimistic t-conorms generate pessimistic S-implicators, optimistic t-conorms generate optimistic Simplicators and t-representable t-norms generate i-representable S-implicators.

\subsubsection{Overview}

Table 1. Relationship between the representability of the $\mathrm{t}$-norm and the representability of the implicator.

\begin{tabular}{ccc}
\hline t-norm & S-implicator & R-implicator \\
\hline t-representable & i-representable & none \\
pessimistic & optimistic & optimistic \\
optimistic & pessimistic & none \\
\hline
\end{tabular}

In Table 1, the relationship between the different kinds of representability for t-norms and the representability of the implicators that are generated by these tnorms is shown. Taking into account Proposition 2, t-representable t-norms have a t-representable $\mathcal{N}$-dual t-conorm which generates an i-representable $\mathrm{S}$-implicator. So, in this sense, a t-representable t-norm generates an i-representable S-implicator. Similarly, a pessimistic (optimistic) t-norm generates an optimistic (resp. pessimistic) S-implicator. We see that only the pessimistic t-norms generate an Rimplicator and S-implicator that can both be represented by one of our types of representability. 


\subsection{Evaluation of representation methods}

In Section 3 we have already seen that the class of pessimistic t-norms is the only one which generate both R- and S-implicators that belong to one of the classes of representable implicators which we discussed before. The superiority of the pessimistic t-norms goes even further as we will see below.

\subsubsection{Smets-Magrez axioms}

The Smets-Magrez axioms, a set of natural and commonly imposed criteria for implicators on the unit interval, were extended to $\mathcal{L}^{I}$ by Cornelis et al. ${ }^{12}$ Briefly, an implicator $\mathcal{I}$ on $\mathcal{L}^{I}$ is said to satisfy the Smets-Magrez axioms if for all $x, y, z$ in $L^{I}$

$$
\begin{array}{ll}
\mathcal{I}\left(\mathcal{N}_{\mathcal{I}}(y), \mathcal{N}_{\mathcal{I}}(x)\right)=\mathcal{I}(x, y) & \text { (contrapositivity) } \\
\mathcal{I}(x, \mathcal{I}(y, z))=\mathcal{I}(y, \mathcal{I}(x, z))) & \text { (exchange principle) } \\
\mathcal{I}(x, y)=1_{\mathcal{L}^{I}} \Leftrightarrow x \leq_{L^{I}} y & \text { (confinement principle) } \\
\mathcal{I} \text { is a continuous }\left(L^{I}\right)^{2} \rightarrow L^{I} \text { mapping } & \text { (continuity) }
\end{array}
$$

where $\mathcal{N}_{I}$ is defined by $\mathcal{N}_{\mathcal{I}}(x)=\mathcal{I}\left(x, 0_{\mathcal{L}^{I}}\right)$ for $x$ in $L^{I}$.

It has been shown by Cornelis et al. ${ }^{12}$ that an implicator $\mathcal{I}$ on $\mathcal{L}^{I}$ for which $\mathcal{I}(D, D) \subseteq D$ satisfies all Smets-Magrez axioms if and only if there exists a continuous increasing permutation $\Phi$ on $\mathcal{L}^{I}$ with increasing inverse such that $\mathcal{I}$ is the $\Phi$-transform of $\mathcal{I}_{\mathcal{T}_{W}}$, i.e.,

$$
\mathcal{I}(x, y)=\Phi^{-1}\left(\mathcal{I}_{\mathcal{T}_{W}}(\Phi(x), \Phi(y))\right)
$$

for all $x$ and $y$ in $L^{I}$. It was later shown ${ }^{20}$ that this means that all implicators $\mathcal{I}$ for which $\mathcal{I}(D, D) \subseteq D$ and which satisfy all Smets-Magrez axioms are necessarily of the form of equation (14), i.e. they are optimistic implicators!

\subsubsection{Arithmetical operations}

As many connectives in fuzzy set theory are expressed by means of arithmetical operations $(+,-, \times, \div)$ on $[0,1]$, it is useful to extend them to $\mathcal{L}^{I}$ as well.

Definition 9. (Arithmetical operators on $\left.\mathcal{L}^{I}\right)^{21}$ The addition, subtraction, multiplication and division of two elements $x=\left[x_{1}, x_{2}\right]$ and $y=\left[y_{1}, y_{2}\right]$ in $L^{I}$ can be defined by

$$
\begin{aligned}
& x \ominus_{\mathcal{L}^{I}} y=\left[\min \left(x_{1}+y_{2}, x_{2}+y_{1}\right), x_{2}+y_{2}\right], \\
& x \ominus_{\mathcal{L}^{I}} y=\left[x_{1}-y_{2}, \max \left(x_{1}-y_{1}, x_{2}-y_{2}\right)\right], \\
& x \otimes_{\mathcal{L}^{I}} y=\left[x_{1} y_{1}, \max \left(x_{1} y_{2}, x_{2} y_{1}\right)\right], \\
& x \oslash_{\mathcal{L}^{I}} y=\left[\min \left(\frac{x_{1}}{y_{1}}, \frac{x_{2}}{y_{2}}\right), \frac{x_{2}}{y_{1}}\right], \text { if } y_{1} \neq 0 \neq y_{2} .
\end{aligned}
$$


Note that the result of these operations is not necessarily an element of $\mathcal{L}^{I}$. Using these operations, the Eukasiewicz t-norm $\mathcal{T}_{W}$ on $\mathcal{L}^{I}$ (see Example 3) can be written similarly as $T_{W}:{ }^{21}$ for $x, y$ in $L^{I}$,

$$
\mathcal{T}_{W}(x, y)=\sup \left(0_{\mathcal{L}^{I}}, x \ominus_{\mathcal{L}^{I}}\left(1_{\mathcal{L}^{I}} \ominus_{\mathcal{L}^{I}} y\right)\right) .
$$

Remarkably, also the optimistic t-norm $\mathcal{T}_{W}^{\prime}$ on $\mathcal{L}^{I}$ with representative $T_{W}$ can be written in a similar way as $T_{W}$ : for $x, y \in L^{I}$,

$$
\mathcal{T}_{W}^{\prime}(x, y)=\sup \left(0_{\mathcal{L}^{I}},\left(x \oplus_{\mathcal{L}^{I}} y\right) \ominus_{\mathcal{L}^{I}} 1_{\mathcal{L}^{I}}\right)=\sup \left(0_{\mathcal{L}^{I}}, x \oplus_{\mathcal{L}^{I}}\left(y \ominus_{\mathcal{L}^{I}} 1_{\mathcal{L}^{I}}\right)\right) .
$$

So, both the optimistic and the pessimistic t-norm with representative $T_{W}$ can be written in a similar way as $T_{W}$ itself. While on $([0,1], \leq)$ both expressions are equal to each other, this is not the case on $\mathcal{L}^{I}$.

Similarly, the optimistic and the pessimistic t-norm on $\mathcal{L}^{I}$ with representative the product t-norm $T_{P}$ on $([0,1], \leq)$ can be written in a similar way as $T_{P}$ : for $x, y$ in $L^{I}$,

$$
\begin{aligned}
& \mathcal{T}_{T_{P}}^{p}(x, y)=\mathcal{T}_{P}(x, y)=x \otimes_{\mathcal{L}^{I}} y, \\
& \mathcal{T}_{T_{P}}^{o}(x, y)=x \oslash_{\mathcal{L}^{I}}\left(1_{\mathcal{L}^{I}} \oslash_{\mathcal{L}^{I}} y\right)=y \oslash_{\mathcal{L}^{I}}\left(1_{\mathcal{L}^{I}} \oslash_{\mathcal{L}^{I}} x\right) .
\end{aligned}
$$

Again, the two formulas are equal to each other on $([0,1], \leq)$, but not on $\mathcal{L}^{I}$.

\subsubsection{A general class of $t$-(co)norms}

In Section 3.2.2 we have seen that t-representable t-norms and pessimistic t-norms are special cases of the class of t-norms $\mathcal{T}_{T, t}$ defined by, for all $x, y$ in $L^{I}$,

$$
\mathcal{T}_{T, t}(x, y)=\left[T\left(x_{1}, y_{1}\right), \max \left(T\left(t, T\left(x_{2}, y_{2}\right)\right), T\left(x_{1}, y_{2}\right), T\left(x_{2}, y_{1}\right)\right)\right],
$$

and that the optimistic t-norms $\mathcal{T}_{T}^{o}$ do not belong to this class as soon as $T \neq \min$.

Other attempts to generalize the pessimistic or optimistic t-norms however give no interesting results. For instance, a symmetrically shaped formula given by, for $x, y$ in $L^{I}$,

$$
\begin{aligned}
\mathcal{M}_{T, t}(x, y) & =\left[\min \left(T\left(t, T\left(x_{1}, y_{1}\right)\right), T\left(x_{1}, y_{2}\right), T\left(x_{2}, y_{1}\right)\right), T\left(x_{2}, y_{2}\right)\right] \\
& =\left[T\left(t, T\left(x_{1}, y_{1}\right)\right), T\left(x_{2}, y_{2}\right)\right] .
\end{aligned}
$$

does not yield a t-norm as soon as $t<1$. So the class of optimistic t-norms cannot be generalized in a similar way as the pessimistic t-norms.

The formulas for pseudo-t-representable t-(co)norms cannot be extended using two different $\mathrm{t}$-(co)norms on $([0,1], \leq)$, since e.g. $\mathcal{T}(x, y)=\left[T_{1}\left(x_{1}, y_{1}\right)\right.$, $\left.\max \left(T_{2}\left(x_{1}, y_{2}\right), T_{2}\left(x_{2}, y_{1}\right)\right)\right]$ is not associative as soon as $T_{1} \neq T_{2}$. On the other hand, the mixed mapping $\mathcal{M}$, given by, for $x, y$ in $L^{I}$,

$$
\mathcal{M}(x, y)=[\min (T(\square x, \diamond y), T(\diamond x, \square y)), \max (T(\square x, \diamond y), T(\diamond x, \square y))]
$$

is not a t-norm on $\mathcal{L}^{I}$ for any t-norm $T \neq \min$ on $([0,1], \leq)$ because there exist $x_{1}, z_{1} \in[0,1]$ such that $T\left(x_{1}, z_{1}\right)<\min \left(x_{1}, z_{1}\right)$, so $\mathcal{M}\left(\left[x_{1}, 1\right], \mathcal{M}\left(\left[z_{1}, 1\right],[0,1]\right)\right)=$ 
$\mathcal{M}\left(\left[x_{1}, 1\right],\left[0, z_{1}\right]\right)=\left[0, T\left(x_{1}, z_{1}\right)\right] \neq\left[0, \min \left(x_{1}, z_{1}\right)\right]=\mathcal{M}\left(\mathcal{M}\left(\left[x_{1}, 1\right],\left[z_{1}, 1\right]\right),[0,1]\right)$, thus $\mathcal{M}$ is not associative.

From the above it is clear that from the point of view of induced properties the most interesting connectives are the pessimistic t-norms together with their (optimistic) residual implicators.

\section{Conclusion}

In this paper we have discussed several ways of representing negators, t-(co)norms and implicators on $\mathcal{L}^{I}$. The most obvious way to represent these operators is componentwise (for t-(co)norms we obtain the so-called t-representable t-(co)norms); this is the approach of most authors which investigate t-norms and related operators in interval-valued fuzzy set theory. Moreover, some of them ${ }^{8,11}$ even dismiss the idea of non-t-representable t-(co)norms by including t-representability in the definition of t-(co)norms. However it was shown by Deschrijver et al. ${ }^{13}$ that not all t-norms on $\mathcal{L}^{I}$ can be represented that way. Classes of non-t-representable tnorms have been introduced ${ }^{18,20}$ and it is demonstrated that they satisfy more interesting properties, which shows that restricting the definition to representable connectives is a bad choice. In this paper we developed a comprehensive typology of representation methods for connectives in interval-valued fuzzy set theory. We have pointed out that one prototypical form of non-representable connectives, namely the pessimistic t-(co)norms and their respective $\mathrm{S}$ - and R-implicators, have the highest number of desirable properties. This observation is very important to the development of future applications of interval-valued fuzzy set theory.

\section{Acknowledgment}

The authors would like to thank the anonymous referee for useful comments. Chris Cornelis would like to thank the Research Foundation - Flanders for supporting his research.

\section{References}

1. L. A. Zadeh, Fuzzy sets, Information and Control 8 (1965) 338-353.

2. J. Goguen, L-fuzzy sets, Journal Math. Anal. Appl. 18 (1967) 145-174.

3. G. Gargov, Knowledge, uncertainty and ignorance in logic: Bilattices and beyond, Journal of Applied Non-Classical Logics 9 (1999) 195-283.

4. R. John, Type 2 fuzzy sets: an appraisal of theory and applications, Int. J. Uncertainty, Fuzziness and Knowledge-Based Systems 6 (1998) 563-576.

5. K. T. Atanassov, Intuitionistic fuzzy sets, (1983), VII ITKR's Session, Sofia (deposed in Central Sci.-Technical Library of Bulg. Acad. of Sci., 1697/84) (in Bulgarian).

6. O. Arieli, C. Cornelis, G. Deschrijver and E. E. Kerre, Uncertainty modeling by bilattice-based squares and triangles, IEEE Transactions on Fuzzy Systems In press.

7. R. Sambuc, Fonctions $\Phi$-floues. Application à l'aide au diagnostic en pathologie thyroidienne, (1975), Ph.D. Thesis, University of Marseille. 
8. M. Gehrke, C. Walker and E. Walker, Some comments on interval-valued fuzzy sets, International Journal of Intelligent Systems 11 (1996) 751-759.

9. J. M. Mendel, Uncertain rule-based fuzzy logic systems (Prentice Hall PTR, Upper Saddle River, New Jersey, 2001).

10. K. T. Atanassov and G. Gargov, Interval-valued intuitionistic fuzzy sets, Fuzzy Sets and Systems 31 (1989) 343-349.

11. S. Jenei, A more efficient method for defining fuzzy connectives, Fuzzy Sets and Systems 90 (1997) 25-35.

12. C. Cornelis, G. Deschrijver and E. E. Kerre, Implication in intuitionistic and intervalvalued fuzzy set theory: Construction, classification, application, International Journal of Approximate Reasoning 35 (2004) 55-95.

13. G. Deschrijver, C. Cornelis and E. E. Kerre, On the representation of intuitionistic fuzzy t-norms and t-conorms, IEEE Transactions on Fuzzy Systems 12 (2004) 45-61.

14. P. Hajek, Metamathematics of Fuzzy Logic (Kluwer Academic Publishers, Dordrecht, 1998).

15. K. T. Atanassov, More on intuitionistic fuzzy sets, Fuzzy Sets and Systems 33 (1989) $37-46$.

16. C. Cornelis, G. Deschrijver and E. E. Kerre, Classification of intuitionistic fuzzy implicators: an algebraic approach, in Proceedings of 6th Joint Conference on Information Sciences, eds. H. J. Caulfield, S. Chen, H. Chen, R. Duro, V. Honavar, E. E. Kerre, M. Lu, M. G. Romay, T. K. Shih, D. Ventura, P. P. Wang and Y. Yang (2002), pp. 105-108.

17. G. Deschrijver, C. Cornelis and E. E. Kerre, Intuitionistic fuzzy connectives revisited, in Proceedings of 9th International Conference on Information Processing and Management of Uncertainty in Knowledge-Based Systems (2002), pp. 1839-1844.

18. G. Deschrijver and E. E. Kerre, Classes of intuitionistic fuzzy t-norms satisfying the residuation principle, International Journal of Uncertainty, Fuzziness and KnowledgeBased Systems 11 (2003) 691-709.

19. M. Baczyński, On some properties of intuitionistic fuzzy implications, in Proceedings of the Third International Conference in Fuzzy Logic and Technology, eds. M. Wagenknecht and R. Hampel (2003), pp. 168-171.

20. G. Deschrijver and E. E. Kerre, Implicators based on binary aggregation operators on $L^{*}$, Fuzzy Sets and Systems 153 (2005) 229-248.

21. G. Deschrijver, Arithmetic operators in interval-valued fuzzy set theory, in press. 